Address for Correspondence: Dr. Guo-Jun Yang,

Department of Cardiovascular, Affiliated Hospital of Ningbo University School of Medicine, Ningbo 315020,

Zhejiang Province, China.

E-mail: yangguojun@medmail.com.cn

\section{Access this article online}

Website:

www.intern-med.com

DOI:

10.1515/jtim-2013-0008

Quick Response Code:

\title{
Antiplatelet therapy in patients with hypertension
}

\author{
Guo-Jun Yang
}

Department of Cardiovascular, Affiliated Hospital of Ningbo University School of Medicine, Ningbo 315020, Zhejiang Province, China

\section{ABSTRACT}

This paper overviewed theoretical basis, clinical evidence and clinical practice principles of antiplatelet therapy in patients with hypertension to draw attention to take appropriate risk stratification, rigorous screening for bleeding cases and to administer aspirin with appropriate dose in daily clinical practice.

Key words: aspirin, hypertension, safety

\section{INTRODUCTION}

Hypertension is an important global public health issue with high incidence and mutilation rate. In United States and European countries, the incidence and mortality rate have been gradually decreased year by year due to decades of untiring efforts, especially the effective implementation of primary prevention measures; whereas the prevalence of hypertension in China has increased substantially in recent years, marked by increased incidence and mutilation rate of apoplexia. ${ }^{[1]}$ Therefore, it is an urgent to reasonably develop and effectively implement primary prevention to contain the prevalence of cardiovascular disease in China.

Indeed, for hypertension itself, to effectively reduce blood pressure is the most effective therapy for prevention and treatment of cardiovascular events. Numerous data have suggested that as long as the systolic pressure is decreased by $10-12 \mathrm{mmHg}$ $(1 \mathrm{mmHg}=0.133 \mathrm{kPa})$ or diastolic pressure decreased by $5-6 \mathrm{mmHg}$, the risks of apoplexy, myocardial infarction and congestive heart failure would be decreased by $35-40 \%, 20-25 \%$ and $50 \%$, respectively. However, some data also indicated that even if the blood pressure were reduced to the standard level, the incidence rates of coronary heart disease and apoplexia in hypertensive patients would remain significantly higher (doubled with coronary heart disease and 3 times with apoplexia), compared with the subjects with normal blood pressure and matched age. ${ }^{[2,3]}$ Apparently, antihypertensive therapy alone is not enough to effectively prevent the cardiovascular events in hypertensive patients; and primary prevention strategies, including the measures of antiplatelet and statin treatment, should be conducted with reference to current effective measures in available clinical trials on basis of the pathogenesis of hypertension targeted at multiple risk factors,. The rationales, clinical trial evidence and the principle of future clinical practice for antiplatelet treatment in hypertensive patients are summarized as follows.

\section{RATIONALES OF ANTIPLATELET TREATMENT IN HYPERTENSIVE PATIENTS}

Hypertension is clinically characterized by increased pressure of arterial vascular wall and it is an essentially vascular lesion. The persistence and progress of hypertension can result in systemic arteriole hyaline degeneration, middle layer smooth muscle cell proliferation, vascular wall thickening 
and luminal stenosis, i.e., vascular wall remodeling. Meanwhile, hypertension can promote the formation and development of atherosclerosis in large and mediumsized arteries. Therefore, the presence of small vessel remodeling was the pathological basis for hypertension which causes cardiovascular events and results in the damages of target organs such as heart, brain and kidney; furthermore, the presence of pathological changes of onset and development of atherosclerosis in large and mediumsized arteries can be induced by hypertension, which triggers coronary and cerebrovascular events. Various basic studies showed that the adhesion, activation and aggregation of platelet on vascular wall and the release of various cytokines and inflammatory factors participated in the occurrence and development of atherosclerosis. First, various risk factors can damage vascular endothelium, platelets which adhered to the injured vascular endothelium and released thromboxane $A_{2}$, platelet-derived growth factor, transforming growth factor-B and plasminogen activator inhibitor, etc., which further induced migration and proliferation of smooth muscle cells and endothelial injury and participated in the occurrence and development of atherosclerosis. ${ }^{[4]}$ Subsequently, the unstableness of plaque during the progress of atherosclerosis was the most important pathophysiological mechanism of cardiovascular and cerebrovascular events. Recent studies have shown that cell chemokines released by platelet further induced monocytes, macrophages and endotheliocytes to secrete tumor necrosis factor and cell chemokine, simultaneously, these factors acted on respective receptors on the surface of platelet to promote the secretion of cell chemokines and inflammatory factors and therefore participated in the formation of unstable plague, i.e., the vicious cycle formed between platelet and inflammatory cells was one of the important mechanisms for the formation of unstable plague. ${ }^{[5,6]}$ Finally, the unstable plague further activated the formation of white thrombus from platelet and then activated clotting system to form large red thrombus and block the vessels, resulting in the occurrence of clinical acute cardiovascular and cerebrovascular events. It is not difficult to understand that platelets participate in the full course from formation of atherosclerosis to the occurrence of acute cardiovascular and cerebrovascular events. Thus, the antiplatelet therapy has strong rationales in treatment of hypertension.

\section{CLINICAL TRIAL EVIDENCE OF ANTIPLATELET THERAPY IN HYPERTENSIVE PATIENTS}

A total of 18790 hypertensive patients from 26 countries were involved in the hypertension optimal treatment (HOT) study in 1998, ${ }^{[7]}$ of which 9399 cases received low-dose treatment of aspirin $75 \mathrm{mg} / \mathrm{d}$ in combination with continuous antihypertensive treatment following diastolic pressure $<90 \mathrm{mmHg}$ and 9391 cases received placebo treatment as the control group with continuous antihypertensive treatment following diastolic pressure reduced to $<90 \mathrm{mmHg}$. Compared with control group, low-dose of aspirin reduced main cardiovascular events and myocardial infarction events by $15 \%(P<0.03)$ and $36 \%(P<0.002)$, respectively. Subgroup analysis in HOT study suggested that with regards to two groups of patients with serum creatinine $>114.9 \mu \mathrm{mol} / \mathrm{L}$ and systolic pressure $>180 \mathrm{mmHg}$ or diastolic pressure $>107 \mathrm{mmHg}$, low-dose of aspirin still showed cardiovascular benefits which significantly outweighed the risk of bleeding. For safety consideration, the administration of aspirin to patients without controlled blood pressure was not recommended in the current guidance; however, the subgroup results at least indicated that blood pressure was not an absolute contraindication for the administration of aspirin.

Nearly 40,000 female health care staff over 45 years old in USA were enrolled in a women health study (WHS) conducted in $2005^{[8]}$ and received $100 \mathrm{mg} / \mathrm{d}$ aspirin or placebo. The results suggested that there was a $17 \%$ reduction in the risk of stroke in the aspirin group, when compared with the placebo group, owing to a $24 \%$ reduction in the risk of ischemic stroke. However, in the group of elderly females over 65-year-old, the risks of total cardiovascular and cerebrovascular events, myocardial infarction and ischemic stroke were reduced by $26 \%(P=0.008), 34 \%(P$ $=0.04)$ and $30 \%(P=0.05)$, respectively. Apart from the elderly group, the total stroke in hypertension subgroup was decreased by $24 \%(P=0.04)$, ischemic stroke by $27 \%$ $(P=0.02)$, the benefit significantly outweighed that of the population without hypertension. The 10-year cardiovascular risk in WHS included population was $2.3 \%$, which was really low-risk population. Therefore, the benefit of aspirin may have been underestimated, whereas the risk of bleeding was correspondingly overestimated. Even so, the overall benefit of aspirin in WHS significantly outweighed its risk of bleeding.

\section{MAIN POINTS NEED TO BE GRASPED IN CLINICAL PRACTICE}

As mentioned above, the benefit of primary prevention with aspirin, including the cardiovascular benefit of primary prevention in hypertensive patients was apparent; however, we must face the fact that aspirin also induced or aggravated alimentary tract hemorrhage at the same time. Although the results of previous primary prevention trials all suggested that the benefit of aspirin outweighed its risk of bleeding, the public still have misgivings. Therefore, 
to achieve the significant outweighing of benefit against bleeding risk is the principle that must be followed in the primary prevention practice of aspirin. The following points are essential for effective implementation of this principle in clinical practice.

\section{Accurately management of the risk stratification} The results of previous aspirin primary prevention trials $\mathrm{s}^{[7-12]}$ suggested that the benefit outweighed bleeding risk in the aspect of cardiovascular and cerebrovascular events in the population with a 10 -year cardiovascular risk of over $6 \%$, whereas the benefit was equivalent to or lower than the bleeding risk when it was below the risk stratification. Therefore, the primary prevention measures in hypertensive patients with aspirin were clearly defined in recent years by the relevant academic organizations both at home and abroad. European Society of Hypertension/European Society of Cardiology (ESH/ESC 2003) recommended: ${ }^{[13]}$ Among the hypertensive patients without cardiovascular diseases, low-dose aspirin was recommended for these with moderate increase of creatinine, $>50$ years old with moderate increase of risk for heart disease, or with high blood pressure baseline. Although this recommendation was modified by ESH/ESC in 2009, it still persisted to recommend the administration of aspirin in hypertensive patients without cardiovascular diseases. ${ }^{[14]}$ American Hypertension Guidelines (JNC-7) ${ }^{[15]}$ believed that the administration of aspirin should be considered for hypertensive patients with controlling blood pressure. Chinese experts had consensus on primary prevention of cardiovascular diseases in $2009^{[16]}$ and specifically stated that the administration of aspirin should be considered for hypertensive patients with stable controlled blood pressure (<150/90 mmHg), accompanied with a high-risk factor, i.e., age $>50$ years old, accompanied with injury of target organs and diabetes. The latest guideline ${ }^{[17]}$ was published by US Preventive Services Task Force in 2009, males aged from 45 to 79 years old and females aged from 55 to 79 years old were encouraged to receive low-dose aspirin as primary prevention measure, whereas the identification of risk stratification was not fixed. The 10 -year cardiovascular risk for different age period from 45 to 79 years old was ranged from $>4 \%$ to $>12 \%$, i.e., for young subjects, the benefit outweighed bleeding risk when their cardiovascular risk stratification was low; while with the increase of age, the requirements of risk stratification will be also increased if the therapeutic effect of benefit outweighing bleeding risk was desired to be achieved. This should be reasonable risk stratification, but the clinical practice is difficult to handle. The recommendation defined by China experts that the population with a 10 -year cardiovascular risk $>10 \%$ should receive aspirin was maneuverable. With regards to hypertensive patients, their 10-year cardiovascular risk was $>6 \%$ if they were accompanied with one of the other risk factors including age $>50$ years old; while the risk was $>10 \%$ if they were accompanied with two of the other risk factors. It was not difficult to conclude from the above mentioned guideline that the administration of lowdose aspirin was recommended for the population with a 10 -year cardiovascular risk $>10 \%$; while the administration of aspirin can be considered after carefully weighing the merits and demerits for the population with a risk between $6 \%$ and $10 \%$; however, the administration of aspirin was not recommended for the population with a 10 -year risk $<5 \%$. The reason for untiring risk stratification determinations was that accurate risk stratification was the key to ensure the outweighing of aspirin benefit against bleeding risk.

\section{Strict screening of bleeding-prone population}

Besides accurate risk stratification, strict screening of bleeding-prone population is another important aspect to ensure the outweighing of aspirin benefit against bleeding risk. Expert Consensus Document on Reducing the Gastrointestinal risk of Antiplatelet Therapy and NSAID Use, published by American College of Cardiology, American Heart Association and American College of Gastroenterology jointly in 2008, clearly stated that ${ }^{[18]}$ for patients with a history of ulcers, gastrointestinal bleeding, dual antiplatelet therapy or combination therapy with anticoagulants such as warfarin, proton pump inhibitors should be added if antiplatelet therapy was necessary, which is equally applicable for the patients with any two simultaneous cases of dyspepsia or gastroesophageal reflux symptoms, age $>65$ years old or the administration of corticosteroids. The reasonability and feasibility of the combination of aspirin primary prevention and proton pump inhibitors therapy is still under investigation; however, the recommendation mentioned above of adding proton pump inhibitors may be not suitable for aspirin primary prevention in most hypertensive patients, while it is very necessary to screen out the bleeding-prone highrisk population for conduct reasonable therapy and then perform aspirin therapy.

\section{Correct handling of aspirin dose}

The evidences from basic studies of antiplatelet therapy and primary prevention clinical trials suggested that the antiplatelet effect and beneficial effect of aspirin was not improved with the increase of dose, whereas the occurrence of alimentary tract hemorrhage was positively correlated to dose. ${ }^{[1]]}$ Therefore, the recommended dosage in international guideline was $75-150 \mathrm{mg} / \mathrm{d}$ or $81-162 \mathrm{mg} / \mathrm{d}, 75-100 \mathrm{mg} / \mathrm{d}$ aspirin was recommended for the hypertensive population with indications by Chinese Expert Consensus (Clinical application suggestions of aspirin in atherosclerotic cardiovascular disease) in 2005 to perform primary prevention treatment. ${ }^{[20]}$ 


\section{SUMMARY}

To sum up, single antihypertensive measures were not sufficient to effectively prevent the risk of cardiovascular events in hypertensive patients. The hazard extent of simultaneously concomitant of other risk factors in 10-year cardiovascular risk should be comprehensively evaluated and specific comprehensive therapy should be conducted to significantly reduce the incidence rate; antiplatelet therapy was one of the effective auxiliary therapies. The primary prevention principle of aspirin administration is similar to that in other high-risk population, i.e., the outweighing of benefit against risk was the basic principle of medication. In clinical practice, the accurate handling of the above mentioned three points was an important guarantee to effectively implement this principle in practice and its clinical benefit. It is believed that with the constant emphasis on primary prevention and the implementation of effective measures, the tendency of increased incidence rate of cardiovascular disease in China can be certainly and effectively controlled.

\section{REFERENCES}

1. National Cardiovascular Disease Center. Chinese Cardiovascular Disease Report 2008-2009. Beijing: Encyclopedia of China Publishing House; 2010. p. 1.

2. Vasan RS, Beiser A, Seshadri S, Larson MG, Kannel WB, D'Agostino $\mathrm{RB}$, et al. Residual lifetime risk for developing hypertension in middle-aged women and men: The Framingham Heart Study. JAMA 2002;287:1003-10.

3. Junga K, Merlo J, Gullberg B, Bog-Hansen E, Rastam L, Lindblad U. Residual risk for acute stroke in patients with type 2 diabetes and hypertension in primary care: Skaraborg hypertension and diabetes project. Diabetes Obes Metab 2006;8:492-500.

4. Ross R. Atherosclerosis - An inflammatory disease. N Engl J Med 1999;340:115-26.

5. Aukrust P, Waehre T, Damås JK, Gullestad L, Solum NO. Inflammatory role of platelets in acute coronary syndromes. Heart 2001;86:605-6.

6. May AE, Seizer P, Gawaz M. Platelets: Inflammatory firebugs of vascular walls. Arterioscler Thromb Vasc Biol 2008;28:s5-10.

7. Hansson L, Zanchetti A, Carruthers SG, Dahlöf B, Elmfeldt D, Julius S, et al. Effects of intensive blood-pressure lowering and low-dose aspirin in patients with hypertension: Principal results of the hypertension optimal treatment (HOT) randomised trial. HOT Study Group. Lancet 1998;351:1755-62.

8. Ridker PM, Cook NR, Lee IM, Gordon D, Gaziano JM, Manson $\mathrm{JE}$, et al. A randomized trial of low-dose aspirin in the primary prevention of cardiovascular disease in women. N Engl J Med 2005;352:1293-304

9. Peto R, Gray R, Collins R, Wheatley K, Hennekens C, Jamrozik K, et al. Randomised trial of prophylactic daily aspirin in British male doctors. Br Med J (Clin Res Ed) 1988;296:313-6.

10. Final report on the aspirin component of the ongoing Physicians' Health Study. Steering Committee of the Physicians' Health Study Research Group. N Engl J Med 1989;321:129-35.

11. Thrombosis Prevention Trial: Randomised trial of low-intensity oral anticoagulation with warfarin and low-dose aspirin in the primary prevention of ischaemic heart disease in men at increased risk. The Medical Research Council's General Practice Research Framework. Lancet 1998;351:233-41.

12. de Gaetano G, Collaborative Group of the Primary Prevention Project. Low-dose aspirin and vitamin $\mathrm{E}$ in people at cardiovascular risk: A randomised trial in general practice. Collaborative Group of the Primary Prevention Project. Lancet 2001;357:89-95.

13. European Society of Hypertension-European Society of Cardiology Guidelines Committee. 2003 European Society of HypertensionEuropean Society of Cardiology guidelines for the management of arterial hypertension. J Hypertens 2003;21:1011-53.

14. Mancia G, Laurent S, Agabiti-Rosei E, Ambrosioni E, Burnier M, Caulfield MJ, et al. Reappraisal of European guidelines on hypertension management: A European Society of Hypertension Task Force document. J Hypertens 2009;27:2121-58.

15. Chobanian AV, Bakris GL, Black HR, Cushman WC, Green LA, Izzo JL Jr, et al. The Seventh Report of the Joint National Committee on Prevention, Detection, Evaluation, and Treatment of High Blood Pressure: The JNC 7 report. JAMA 2003;289:2560-72.

16. Chinese College of Cardiovascular Physicians of Chinese Medical Association, Editorial Committee of Chinese Journal of Internal Medicine. Chinese experts consensus on primary prevention of cardiovascular diseases. Chin J Intern Med 2010;49:174-89.

17. US Preventive Services Task Force. Aspirin for the prevention of cardiovascular disease: U.S. Preventive Services Task Force recommendation statement. Ann Intern Med 2009;150:396-404.

18. Bhatt DL, Scheiman J, Abraham NS, Antman EM, Chan FK, Furberg CD, et al. ACCF/ACG/AHA 2008 expert consensus document on reducing the gastrointestinal risks of antiplatelet therapy and NSAID use: A report of the American College of Cardiology Foundation Task Force on Clinical Expert Consensus Documents. J Am Coll Cardiol 2008;52:1502-17.

19. Antithrombotic Trialists' (ATT) Collaboration, Baigent C, Blackwell L, Collins R, Emberson J, Godwin J, et al. Aspirin in the primary and secondary prevention of vascular disease: Collaborative metaanalysis of individual participant data from randomised trials. Lancet 2009;373:1849-60.

20. Chinese College of Cardiovascular Diseases of Chinese Medical Association, Editorial Committee of Chinese Journal of Cardiology. Clinical application of aspirin in atherosclerotic cardiovascular diseases. Chinese Experts Consensus (2005). Chin J Cardiol 2006;34:281-4.

How to cite this article: Yang GJ. Antiplatelet therapy in patients with hypertension. J Transl Intern Med 2013; 1(1): 28-31.

Source of Support: Nil, Conflict of Interest: None declared 